\title{
SUBSTRATO E ADUBAÇÃO FOSFATADA PARA A PRODUÇÃO DE MUDAS CLONAIS DE CACAU ${ }^{(1)}$
}

\author{
José Olimpio de Souza Júnior ${ }^{(2)}$, Quirino Augusto de Camargo \\ Carmello $^{(3)} \&$ George Andrade Sodré $(2,4)$
}

\begin{abstract}
RESUMO
Para avaliar o efeito de substratos e da adubação fosfatada sobre a produção de mudas de cacaueiro, bem como definir doses recomendáveis e nível crítico foliar de $P$, fez-se um experimento fatorial 5 x $5+1$ : cinco substratos (misturas de fibra de coco (FC) e Plantmax florestal estaca ${ }^{\circledR}$ ), cinco doses de P no plantio (de 0 a $800 \mathrm{mg} \mathrm{dm}^{-3}$ ) e um tratamento adicional ( $\mathrm{P}$ aplicado aos 30 dias). As parcelas iniciais e úteis continham, respectivamente, 27 estacas e 12 mudas (uma estaca/ muda por tubete). A partir do $62^{\circ}$ dia, aplicaram-se adubações semanais com $\mathrm{N} \mathrm{e}$ $\mathrm{K}$, e aos 120 dias, com $\mathrm{P}\left(20 \mathrm{mg} \mathrm{dm}^{-3}\right)$, em todos os tratamentos. Aos 150 dias foram avaliados: diâmetro, altura, área foliar, massa de matéria seca da parte aérea e das raízes (finas e grossas), concentração e conteúdo de nutrientes na planta. A mortalidade das mudas não foi influenciada pelos tratamentos. A adubação com $\mathbf{P}$ em cobertura aumentou sua disponibilidade e sua absorção, mas não o crescimento das mudas. As variáveis biométricas e nutricionais responderam aos tratamentos, sendo os melhores resultados obtidos com 30 a $55 \%$ de FC e doses de P entre 136 e $275 \mathrm{mg} \mathrm{dm}^{-3}$. O nível crítico foliar de $\mathrm{P}$ foi de $1,75 \mathrm{~g} \mathrm{~kg}^{-1}$.
\end{abstract}

Termos de indexação: Theobroma cacao, nutrição, nível crítico, cultivo sem solo.

\section{SUMMARY: POTTING MIX AND PHOSPHATE FERTILIZATION FOR PRODUCTION OF ROOTED COCOA CUTTINGS}

The effect of potting mix and phosphate fertilization on the production of rooted cocoa tree cuttings was evaluated and recommended rates and critical foliar P levels were determined

\footnotetext{
(1) Recebido para publicação em março de 2009 e aprovado em outubro de 2010.

(2) Departamento de Ciências Agrárias e Ambientais, Universidade Estadual de Santa Cruz - UESC. km 16, Rod. Ilhéus/Itabuna, CEP 45652-000 Ilhéus (BA). E-mail: olimpio@uesc.br

(3) Departamento de Ciência do Solo, Escola Superior de Agricultura "Luiz de Queiroz", Universidade de São Paulo - ESALQ/ USP. Av. Pádua Dias 11, Cx. Postal 09, CEP 13418-900 Piracicaba (SP). E-mail: qaccarme@esalq.usp.br

(4) Comissão Executiva do Plano da Lavoura Cacaueira, Centro de Pesquisa do Cacau - CEPLAC/CEPEC. km 22, Rod. Ilhéus/ Itabuna, CEP 45600-000 Itabuna (BA). E-mail: sodre@cepec.gov.br
} 


\begin{abstract}
based on a factorial $5 \times 5+1$ experiment: five potting medium (coconut fiber - CF and Plantmax $\left.{ }^{\circledR} \mathrm{mix}\right)$, five $P$ rates at planting $\left(0\right.$ to $\left.800 \mathrm{mg} \mathrm{dm}^{-3}\right)$ and an additional $P$ treatment (applied on the $30^{\text {th }}$ day). Each plot contained 27 cuttings, of which 12 rooted cuttings were evaluated. From the $62^{\text {nd }}$ day onwards, $N$ and K fertilization was weekly applied as well as a $P$ fertilization on the $120^{\text {th }}$ day $\left(20 \mathrm{mg} \mathrm{dm}^{-3}\right)$, to all treatments. The diameter, height, leaf area, shoot and root (fine and thick) dry matter, nutrient concentration and content in plants were evaluated on the $150^{\text {th }}$ day. Cutting mortality was not influenced by the treatments. Phosphorus top dressing increased $P$ assimilation and absorption, but not the growth of the rooted cuttings. The biometric and nutrition variables responded to treatments; best results were obtained with $30-55 \%$ of $C F$ and $P$ rates between 136 and $275 \mathrm{mg} \mathrm{dm}^{-3}$. The foliar critical level of $P$ was $1.75 \mathrm{~g} \mathrm{~kg}^{-1}$.
\end{abstract}

Index terms: Theobroma cacao, plant nutrition, critical level, soilless cultivation.

\section{INTRODUÇÃO}

A cacauicultura do sudeste da Bahia vem atravessando nos últimos anos uma crise de produção, que foi agravada a partir de 1989, com o surgimento e a disseminação rápida da doença vassoura-de-bruxa. O cacaueiro (Theobroma cacao L.) ainda é a principal cultura agrícola dessa região e representa a principal receita da economia de aproximadamente 100 municípios.

Para auxiliar a recuperação e a renovação da cacauicultura dessa região, foi criado o Instituto Biofábrica de Cacau (IBC), que tem como principal função multiplicar e distribuir materiais botânicos de cacau com potencial produtivo elevado e tolerância à vassoura-de-bruxa, selecionados pela Comissão Executiva do Plano da Lavoura Cacaueira (CEPLAC) e por produtores. Esse material tem sido usado para substituir as plantas enfermas, adensar áreas e para o plantio das novas lavouras.

As mudas de cacau, oriundas do processo atual de produção, não vêm sendo disponibilizadas aos produtores em condições consideradas ótimas com relação aos aspectos nutricionais; dentre esses problemas, destacam-se a toxidez de Fe (Marrocos \& Sodré, 2004) e a necessidade de calibração de P (Souza Jr. et al., 2008). A fonte desse excesso de Fe pode ser o Plantmax ${ }^{\circledR}$ (Pmax), que juntamente com a fibra de coco (FC), na proporção volumétrica de 1:1, compõem o substrato utilizado no IBC, para a produção de mudas de cacau (Marrocos \& Sodré, 2004).

É comum o uso de fertilizantes fosfatados em adubação pré-plantio em substratos (Williams \& Nelson, 1996; Bataglia \& Furlani, 2004), porém as doses recomendadas são bastante variáveis. Para produção de mudas de espécies florestais, como Eucalyptus e Pinus, em tubetes, os manuais nacionais recomendam doses de $\mathrm{P}$ que variam de $130 \mathrm{mg} \mathrm{dm}^{-3}$ (Gonçalves et al., 1997) a $655 \mathrm{mg} \mathrm{dm}^{-3}$ (Barros \& Novais, 1999).

O objetivo deste trabalho foi avaliar a sobrevivência, o crescimento e a nutrição de mudas de cacaueiro cultivadas em substratos formados por misturas de fibra de coco e Plantmax ${ }^{\circledR}$ e adubadas com superfosfato triplo. Avaliou-se também o efeito da época de fertilização fosfatada, bem como foi determinado o substrato mais eficiente e econômico, as doses recomendáveis e o nível crítico foliar de $\mathrm{P}$.

\section{MATERIAL E MÉTODOS}

O experimento foi instalado em julho de 2005, em viveiro de produção de mudas, com as laterais e o teto de tela plástica preta com $50 \%$ de sombreamento, no Instituto Biofábrica de Cacau (IBC), em Ilhéus, Bahia.

Foi utilizado um fatorial $5 \times 5+1$ (cinco substratos, cinco doses de $\mathrm{P}$ no plantio e um tratamento adicional), em delineamento de blocos ao acaso, com três repetições. Os substratos utilizados foram cinco proporções (20:80; 35:65; 50:50; 65:35 e 80:20 \%, em volume) dos substratos fibra de coco (FC) e Plantmax florestal estaca ${ }^{\circledR}$ (Pmax - substrato comercial, composto de casca de pínus compostada, vermiculita expandida, carvão granulado e turfa).

As doses de P no plantio foram: 0; 100; 200; $400 \mathrm{e}$ $800 \mathrm{mg} \mathrm{dm}^{-3}$, na forma de superfosfato triplo (SFT) moído, aplicado e homogeneizado em um volume de substrato equivalente ao do tubete. No tratamento adicional utilizou-se o substrato com $50 \%$ de FC e $50 \%$ de Pmax, e $200 \mathrm{mg} \mathrm{dm}^{-3}$ de $\mathrm{P}$ aplicado em cobertura aos 30 dias após o estaqueamento.

Os substratos originais foram peneirados, em malha de $5 \mathrm{~mm}$, e caracterizados química e fisicamente (Quadros 1 e 2). Foram determinados $\mathrm{pH}$, condutivi-dade eletrolítica (CE) e teor de nutrientes, pelo método extrato em $\mathrm{H}_{2} \mathrm{O}$ 1:1,5 v/v (Sonneveld et al., 1974), CTC e densidade (Brasil, 2006) e curva de retenção de água (De Bood \& Verdonck, 1972). Em amostra seca ao ar e peneirada $(2,0 \mathrm{~mm})$, determinaram-se também os teores disponíveis por métodos de solo, de acordo com Embrapa (1999): resina de troca iônica (P); $\mathrm{KCl}$ 1,0 mol L-1 (Ca, Mg e Al); Mehlich-1 (K); $\mathrm{Ca}\left(\mathrm{H}_{2} \mathrm{PO}_{4}\right)_{2}, 500 \mathrm{mg} \mathrm{L}^{-1}$ de P (S-SO $\left.{ }_{4}^{-2}\right)$; DTPA $(\mathrm{Cu}, \mathrm{Fe}, \mathrm{Mn}$ e $\mathrm{Zn})$ e $\mathrm{CaCl}_{2} 1,25 \mathrm{~g} \mathrm{~L}^{-1}$ a quente (B). Para a análise dos teores totais, procedeu-se à moagem da amostra, seguida por digestão nítricoperclórica, de acordo com Embrapa (1999). 
Quadro 1. Teores de nutrientes solúveis, disponíveis e totais dos substratos fibra de coco (FC) e Plantmax florestal estaca ${ }^{\circledR}(\operatorname{Pmax})$

\begin{tabular}{|c|c|c|c|c|c|c|c|c|c|c|}
\hline Substrato & $\mathbf{P}$ & $\mathbf{S}$ & $\mathbf{K}$ & $\mathbf{C a}$ & Mg & B & $\mathrm{Cu}$ & $\mathbf{F e}$ & Mn & $\mathrm{Zn}$ \\
\hline & \multicolumn{10}{|c|}{$\begin{array}{c}\text { Teor solúvel } \\
\mathrm{mg} \mathrm{L}^{-1}\end{array}$} \\
\hline$P \max$ & 1,5 & 161 & 91 & 115 & 51 & 0,07 & 0,04 & 0,10 & 1,19 & 0,04 \\
\hline \multirow[t]{3}{*}{$\mathrm{FC}$} & 7,9 & 2 & 288 & 1 & 4 & 0,19 & 0,01 & 0,02 & 0,03 & 0,01 \\
\hline & \multicolumn{10}{|c|}{ Teor disponível ${ }^{(2)}$} \\
\hline & \multicolumn{2}{|c|}{$-\mathrm{mg} \mathrm{dm}^{-3} \_$} & $\longrightarrow$ & $\mathrm{ol}_{\mathrm{c}} \mathrm{d}$ & 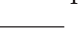 & . & 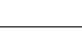 & $\mathrm{mg} \mathrm{dm}^{-3}$ & & - \\
\hline$P \max$ & 203 & 868 & 17 & 129 & 65 & 1,2 & 0,7 & 98 & 38 & 5,0 \\
\hline \multirow[t]{2}{*}{$\mathrm{FC}$} & 16 & 5 & 29 & 3 & 10 & 0,8 & 0,1 & 1 & 2 & 1,0 \\
\hline & \multicolumn{10}{|c|}{ Teor total ${ }^{(3)}$} \\
\hline Pmax & 1,6 & 5,7 & 4,2 & 7,0 & 12,7 & 8 & 21 & 14625 & 207 & 48 \\
\hline $\mathrm{FC}$ & 0,4 & 0,5 & 13,4 & 0,8 & 1,6 & 24 & 2 & 657 & 35 & 17 \\
\hline
\end{tabular}

(1) Teor solúvel: extrato de $\mathrm{H}_{2} \mathrm{O}$ 1:1,5 v/v (Sonneveld et al., 1974). ${ }^{(2)}$ Teor disponível: $\mathrm{P}$ (resina de troca iônica); $\mathrm{S}-\mathrm{SO}{ }_{4}^{-2}\left(\mathrm{Ca}\left(\mathrm{H}_{2} \mathrm{PO}_{4}\right)_{2}\right.$, $500 \mathrm{mg} \mathrm{L}^{-1}$ de P); K (Mehlich-1), Ca e Mg (KCl 1,0 mol L-1); Cu, Fe, Mn e Zn (DTPA) e B ( $\mathrm{H}_{2} \mathrm{O}$ em CaCl ${ }_{2} 1,25$ g L-1 a quente), de acordo com Embrapa (1999). ${ }^{(3)}$ Teor total: digestão nítrico-perclórica, de acordo com Embrapa (1999).

Quadro 2. Análises ${ }^{(1)}$ física e química dos substratos fibra de coco (FC) e Plantmax florestal estaca ${ }^{\circledR}$ (Pmax)

\begin{tabular}{|c|c|c|c|c|c|c|c|c|c|}
\hline Substrato & pH & CE & CTC & DS & PT & EA & AFD & $\mathbf{A T}$ & $\mathrm{AD}$ \\
\hline & & $\mathrm{dS} \mathrm{m}^{-1}$ & $\mathrm{mmol}_{\mathrm{c}} \mathrm{dm}^{-3}$ & $\mathrm{~kg} \mathrm{dm}^{-3}$ & & & $\mathrm{~m}^{3} \mathrm{~m}^{-3}$ & & \\
\hline Pmax & 5,2 & 1,1 & 289 & 0,45 & 0,63 & 0,04 & 0,18 & 0,08 & 0,27 \\
\hline $\mathrm{FC}$ & 5,6 & 0,8 & 34 & 0,06 & 0,75 & 0,12 & 0,33 & 0,06 & 0,39 \\
\hline
\end{tabular}

(1) Métodos: pH e CE (condutividade eletrolítica), Sonneveld et al. (1974); CTC e DS (densidade de material seco), BRASIL (2006); PT (porosidade total), EA (espaço de aeração), AFD (água facilmente disponível), AT (água de tamponamento) e AD (água disponível), obtidos pela curva de retenção de água, de acordo com De Bood \& Verdonck (1972).

No extrato $\mathrm{H}_{2} \mathrm{O}$ 1:1,5 v/v, os elementos foram dosados por espectrometria de emissão atômica (ICPOES). Nos demais extratos, os elementos foram assim dosados: colorimetria (P e B); turbidimetria (S); fotometria de chama (K); e espectrometria de absorção atômica (Ca, $\mathrm{Mg}, \mathrm{Cu}, \mathrm{Fe}, \mathrm{Mn}$ e $\mathrm{Zn}$ ).

A parcela inicial foi formada por uma bandeja com 27 tubetes com $288 \mathrm{~cm}^{3}$ de substrato e uma estaca de cacaueiro do clone PH 16 por tubete. Utilizaram-se estacas apicais semi-herbáceas, de ramos plagiotrópicos, com aproximadamente $18 \mathrm{~cm}$ de comprimento, contendo três folhas, com 1/2 a 2/3 do limbo foliar remanescente. A base das estacas foi tratada com $6 \mathrm{~g} \mathrm{~kg}^{-1}$ de ácido indolbitírico (AIB) em talco; sendo a profundidade de estaqueamento em torno de $6 \mathrm{~cm}$.

A partir do $62^{\circ}$ dia foram feitas adubações semanais com 40 e $20 \mathrm{mg} \mathrm{dm}^{-3}$ de $\mathrm{N}$ e de $\mathrm{K}$, respectivamente, na forma de ureia e cloreto de potássio, num total de 10 adubações. Aos 120 dias, fez-se uma adubação complementar, para todos os tratamentos, com $20 \mathrm{mg} \mathrm{dm}^{-3} \mathrm{de} \mathrm{P}$, na forma de fosfato monoamônico. Os fertilizantes foram aplicados na forma líquida, sendo utilizados 2,0 $\mathrm{mL}$ da solução por tubete.

O controle fitossanitário, no total de nove aplicações durante os 150 dias de cultivo, seguiu o adotado no sistema de produção do IBC, com a utilização de fungicidas, acaricidas e inseticidas, à base de mancozeb, óxido cuproso, endosulfan e methamidophos, com, respectivamente, oito, duas, três e duas aplicações.

A irrigação também foi semelhante à adotada pelo IBC: nebulização intermitente automática. O sistema funcionou das 6 às $18 \mathrm{~h}$, sendo desligado nos períodos com chuva. Utilizaram-se bicos com vazão teórica de $40 \mathrm{~L} \mathrm{~h}^{-1}$ e área irrigada de $4 \mathrm{~m}^{2}$ por bico. Nos primeiros 60 dias, a irrigação foi efetuada a cada 5 min; posteriormente, o número de irrigações foi gradativamente reduzido, até alcançar três irrigações diárias. O tempo de irrigação foi ajustado de acordo com a fase de desenvolvimento das mudas e as condições climáticas.

A sobrevivência das estacas/mudas foi avaliada aos 90, 120 e 150 dias. A parcela útil foi mantida com 12 mudas, sendo as demais plantas identificadas e mantidas como sobressalentes para possível reposição na parcela útil, se necessário.

Aos 150 dias, avaliaram-se: diâmetro da haste principal, com auxílio de paquímetro digital, tomando duas medidas perpendiculares entre si e à distância de aproximadamente $1 \mathrm{~cm}$ do ponto de brotamento; e 
altura da haste principal, medida com régua. A parte aérea das plantas foi colhida (apenas os ramos emitidos), sendo separada em: folha diagnóstica (terceira folha do primeiro lançamento maduro), demais folhas e caule. A área foliar foi medida com auxílio de medidor de área foliar.

O material vegetal da parte aérea (folhas e caule) foi lavado com rápida imersão em: água corrente, solução de detergente neutro $1 \mathrm{~g} \mathrm{~L}^{-1}$, água corrente, solução de $\mathrm{HCl} 30 \mathrm{~mL} \mathrm{~L}^{-1} \mathrm{e}$, finalmente, com duas lavagens com água destilada. Quanto à folha diagnóstica, após a solução de detergente, fez-se a lavagem mecânica, esfregando-se levemente a superfície da folha com chumaço de algodão embebido com a solução de detergente. Optou-se por essa lavagem vigorosa devido ao uso de agrotóxicos que continham nutrientes.

O sistema radicular foi lavado e, após seco, removeuse o substrato que ainda permanecia aderido às raízes, as quais foram visualmente separadas em raízes finas e grossas; o limite médio para separação desses dois grupos foi em torno de $0,4 \mathrm{~mm}$ de diâmetro.

Todas as amostras vegetais foram secas em estufa com circulação forçada de ar a $65^{\circ} \mathrm{C}$, por 3 dias; em seguida, foram pesadas para se obter: massa de matéria seca da parte aérea (MSPA), que foi a soma das matérias secas das folhas e do caule; massa de matéria seca das raízes finas (MSRF), das raízes grossas (MSRG) e das raízes total $(\mathrm{MSRT}=\mathrm{MSRF}+$ MSRG); e massa de matéria seca da planta (MSPL = MSPA + MSRT). Posteriormente, as amostras de folhas e caule foram moídas e analisadas quimicamente, de acordo com Embrapa (1999).

Os resultados foram submetidos à análise de variância, sendo os fatores quantitativos, doses de $\mathrm{Pe}$ proporções de FC no substrato, submetidos à análise de regressão, com coeficientes linear, quadrático ou raiz-quadrático e interação. Foram aceitos os modelos que apresentaram todos os coeficientes significativos a até $10 \%$, pelo teste $\mathrm{F}$, e o maior coeficiente de determinação ajustado.

As doses recomendáveis de $\mathrm{P}$ e os níveis críticos na folha foram calculados para obter $99 \%$ da produção máxima da MSPA, em combinações distintas de FC:Pmax, sendo identificado o custo mínimo entre as combinações dos insumos: substratos (fibra de coco e Plantmax florestal estaca ${ }^{\circledR}$ ) e fertilizante fosfatado.

$\mathrm{O}$ tratamento adicional, $\mathrm{P}$ aplicado em cobertura aos 30 dias, foi analisado por contraste com o tratamento que recebeu a mesma dose de $\mathrm{P}$ no plantio, sendo os contrastes considerados significativos a até $5 \%$ pelo teste $\mathrm{F}$.

\section{RESULTADOS E DISCUSSÃO}

A análise química evidenciou que o Plantmax florestal estaca ${ }^{\circledR}(\operatorname{Pmax})$ é um substrato com maiores teores de elementos solúveis, disponíveis e totais que os da fibra de coco (FC), exceto para K (Quadro 1), sendo um substrato com maior CTC (Quadro 2). Ambos os substratos possuem valores adequados de CE e de pH (Quadro 2), de acordo com classificação proposta por Baumgarten (2002).

No quadro 1, chamam a atenção os teores altos de Fe total e disponível no Pmax, fato também constatado por Marrocos \& Sodré (2004), que também apontam correlação estreita entre as formas totais e solúveis de Fe em substratos utilizados na produção de mudas de cacau, com consequente surgimento de problemas de toxidez desse elemento nas plantas.

O Pmax apresentou baixo espaço de aeração (macroporos) e densidade mais elevada; por sua vez, a FC mostrou ser um substrato pouco denso, mais poroso, com maior espaço de aeração e com maior capacidade de armazenamento de água disponível (Quadro 2), evidenciando ter bons atributos físicos (Abad et al., 2005).

A mortalidade média das mudas aos 90, 120 e 150 dias foi de 30 , 35 e $36 \%$, respectivamente, valores superiores aos encontrados por Faria \& Sacramento (2003) para três clones de cacau tratados com doses de AIB. Essa mortalidade elevada pode estar associada ao período inicial de cultivo (julho e agosto), em que foram observadas precipitações pluviais frequentes e temperaturas mais baixas. A sobrevivência das mudas não foi alterada pelos substratos, pelas doses ou épocas de adubação com $P$.

$\mathrm{A}$ adição de $\mathrm{P}$ aumentou todas as variáveis biométricas avaliadas até o ponto de produção máxima, visto que foram observados efeitos linear e quadrático ou raiz-quadrático significativos do $\mathrm{P}$ (Figura 1a,b,c,e,f), exceto para a MSRF, em que o $\mathrm{P}$ apresentou apenas efeito linear negativo (Figura 1a), ou seja, o fertilizante fosfatado reduziu a produção de raízes finas, fato também constatado por Mackay \& Barber (1985) para densidade de raízes finas em plantas de milho. $\mathrm{O}$ fornecimento de $\mathrm{P}$ favoreceu mais o desenvolvimento da parte aérea que o sistema radicular e, entre as raízes, mais as grossas que as finas (Figura 1d).

A percentagem de $\mathrm{FC}$ no substrato gerou pontos de máxima produção para MSPA, MSPL, diâmetro, altura e área foliar (Figura 1b, c, e, f) e não alterou a MSRF; entretanto, o aumento de FC no substrato incrementou a MSRG e, consequentemente, a MSRT (Figura 1a). Interações significativas entre dose de $\mathrm{P}$ e percentagem de FC foram observadas para MSRT, MSPA, MSPL e área foliar (Figura 1a,b,f), evidenciando que o efeito de $\mathrm{P}$ depende do substrato utilizado.

As equações de regressão selecionadas para altura e área foliar, apesar de significativas, tiveram coeficientes de determinação baixos (Figura1e,f), indicando preditividade baixa desses modelos.

As produções máximas para MSPA e diâmetro foram alcançadas com substrato com $43 \%$ de FC e 

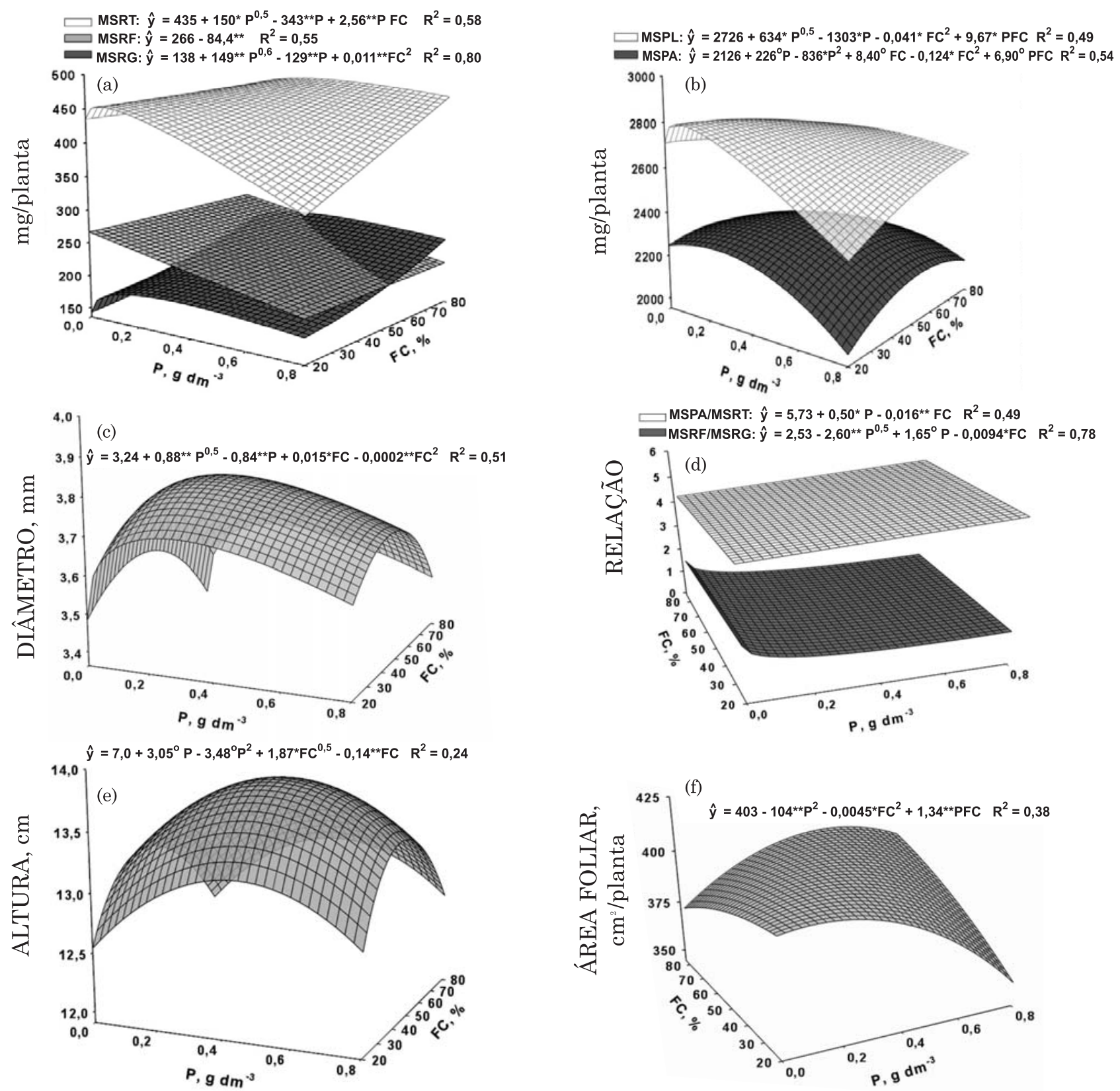

Figura 1. Variáveis biométricas de mudas de cacaueiro, clone PH 16, aos 150 dias, em função da dose de Pe da percentagem de fibra de coco (FC) no substrato: (a) massa de matéria seca das raízes grossas MSRG, raízes finas - MSRF e raízes totais - MSRT; (b) matéria seca da parte aérea - MSPA e da planta - MSPL; (c) diâmetro; (d) relação MSRF / MSRG e MSPA / MSRT; (e) altura; e (f) área foliar. ${ }^{\circ}$, $^{*} \mathrm{e}^{* *}$ : significativo a 10,5 e $1 \%$ pelo teste $F$, respectivamente.

$311 \mathrm{mg} \mathrm{dm}^{-3}$ de $\mathrm{P}$ e $45 \%$ de $\mathrm{FC}$ e $274 \mathrm{mg} \mathrm{dm}^{-3}$ de $\mathrm{P}$, respectivamente. Considerando incrementos de $5 \%$ de cada substrato original na mistura FC:Pmax, podese obter $99 \%$ da produção máxima da MSPA para substrato com teor de FC variando de 30 a $55 \%$ (Quadro 3).

As doses recomendáveis de $\mathrm{P}$ variaram de 136 a $275 \mathrm{mg} \mathrm{dm}^{-3}$ e foram menores para os substratos com proporções de FC:Pmax intermediárias (Quadro 3). A dose de $136 \mathrm{mg} \mathrm{dm}^{-3}$ é semelhante à fertilização de base, de $130 \mathrm{mg} \mathrm{dm}^{-3}$ de $\mathrm{P}$, recomendada por Gonçalves et al. (1997) para a produção de mudas de eucalipto em substrato. Nesse contexto, quando se considera o custo dos insumos, a combinação mais econômica seria para um substrato com $55 \%$ de FC e $45 \%$ de Pmax, adubado com $275 \mathrm{mg} \mathrm{dm}^{-3}$ de P (Quadro 3).

Para cada mistura FC:Pmax foi determinado o nível crítico foliar (Quadro 3), a partir da substituição da dose recomendável e do respectivo teor de FC no substrato na equação da concentração de $\mathrm{P}$ na folha 
Quadro 3. Doses recomendáveis de $\mathrm{P}$ (DRP) para obter 99 \% da produção máxima da matéria seca da parte aérea e níveis críticos (NC) de $P$ na folha, de acordo com a percentagem de fibra de $\operatorname{coco}\left(\right.$ FC) e Plantmax ${ }^{\circledR}$ (Pmax) no substrato, com respectivo custo ${ }^{(1)}$ do substrato adubado com $P$ - Clone PH 16, aos 150 dias

\begin{tabular}{cccc}
\hline FC:Pmax & DRP & Custo $^{(1)}$ & NC na folha \\
\hline$\%, \mathrm{v} / \mathrm{v}$ & $\mathrm{g} \mathrm{m}^{-3}$ & ${\mathrm{R} \$ \mathrm{~m}^{-3}}^{-3}$ & $\mathrm{~g} \mathrm{~kg}^{-1}$ \\
$30: 70$ & 173 & 170 & 1,77 \\
$35: 65$ & 137 & 163 & 1,75 \\
$40: 60$ & 136 & 156 & 1,74 \\
$45: 55$ & 156 & 149 & 1,73 \\
$50: 50$ & 198 & 142 & 1,74 \\
$55: 45$ & 275 & 136 & 1,76
\end{tabular}

(1) Custo dos insumos colocados no Instituto Biofábrica de Cacau, Ilhéus-BA; preços tomados em junho de 2010: Plantmax florestal estaca ${ }^{\circledR}-R \$ 210,00$ por $\mathrm{m}^{3}$; fibra de coco $-\mathrm{R} \$ 73,50$ por $\mathrm{m}^{3 ;}$ e superfosfato triplo $\left(190 \mathrm{~g} \mathrm{~kg}^{-1} \mathrm{de} \mathrm{P}\right)-\mathrm{R} \$ 500,00$ por tonelada. diagnóstica (Figura 2a). Como a variação do nível crítico foliar foi pequena (Quadro 3), pode-se considerar uma concentração média de $1,75 \mathrm{~g} \mathrm{~kg}^{-1}$ de $\mathrm{P}$, valor que é semelhante a $1,8 \mathrm{~g} \mathrm{~kg}^{-1}$, sugerido por Raij et al. (1997), mas inferior a 2,0 $\mathrm{g} \mathrm{kg}^{-1}$, indicado por Malavolta (2006) como adequado para cacaueiros adultos.

De modo geral, observa-se que os modelos para a concentração dos nutrientes na folha diagnóstica (primeiro ramo emitido), em função da dose de SFT e da percentagem de FC no substrato, foram mais bem ajustados que aqueles para conteúdo na parte aérea (Figuras 2 e 3), exceto para $\mathrm{Ca}$ e $\mathrm{Mg}$, sugerindo que os fatores em estudo influenciaram mais a absorção de nutrientes do que a conversão destes em matéria seca e, consequentemente, em quantidade total de nutrientes acumulada pela planta.

$\mathrm{O}$ modelo que apresentou o melhor ajuste, $\mathrm{R}^{2}=$ 0,90, foi o da concentração de P na folha (Figura 2a), nutriente que é uma das variáveis independentes deste trabalho. Contudo, os modelos da concentração de outros nutrientes também mostraram bons ajustes, em especial Zn, N, K, S e Mn (Figura 2), indicando
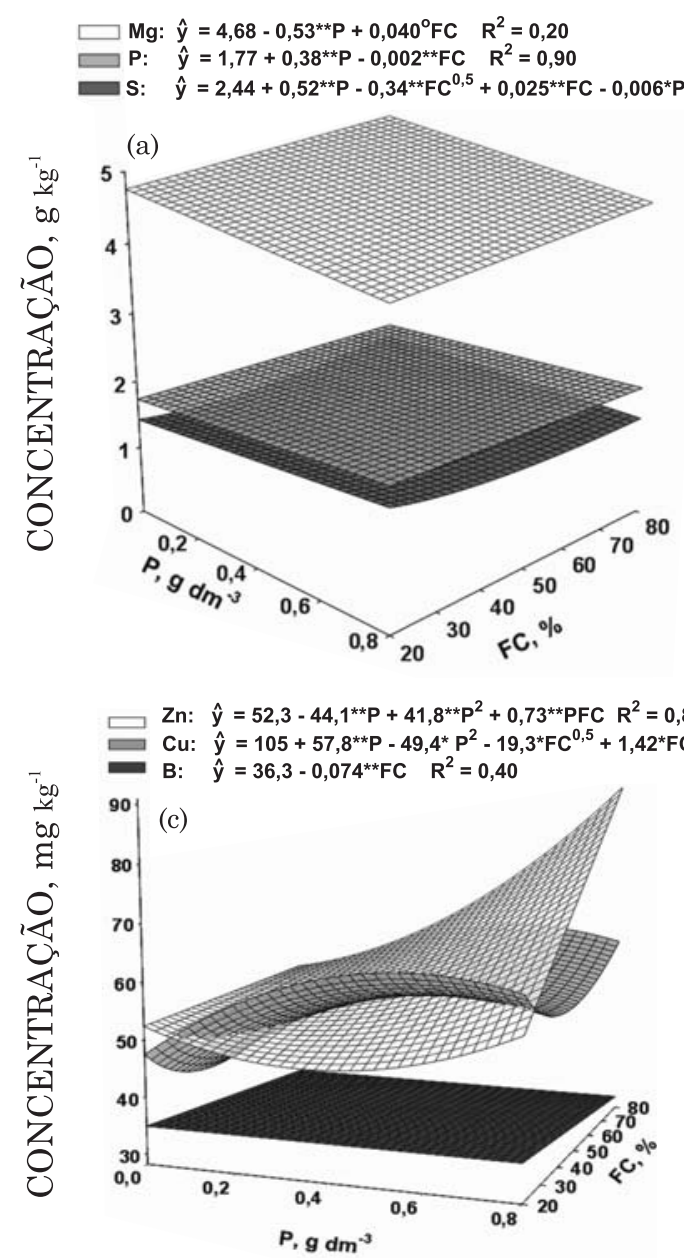
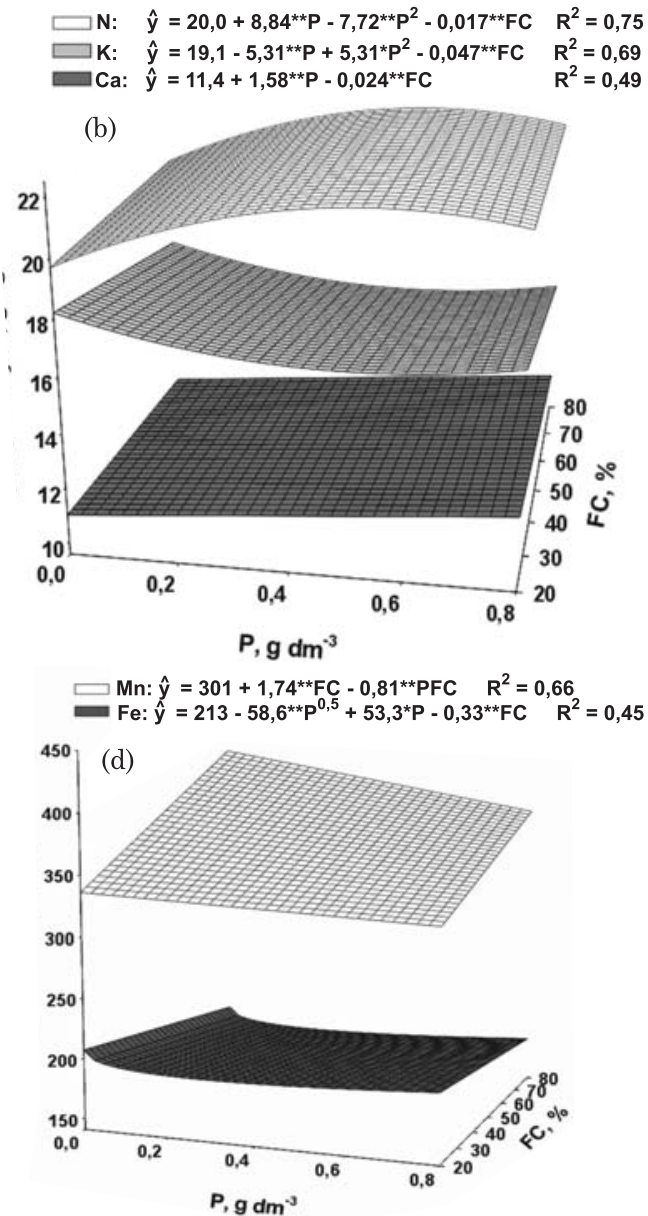

Figura 2. Concentração de nutrientes na folha diagnóstica de mudas de cacaueiro, clone $\mathrm{PH} 16$, aos 150 dias, em função da dose de $\mathrm{P}$ e da percentagem de fibra de coco (FC) no substrato: (a) $\mathrm{Mg}, \mathrm{P}$ e $\mathrm{S}$; (b) $\mathrm{N}$, $\mathrm{K}$ e Ca; (c) $\mathrm{Zn}, \mathrm{Cu}$ e B; e (d) Mn e Fe. ${ }^{\circ},{ }^{*} \mathrm{e}^{* * *}$ : significativo a 10, 5 e $1 \%$ pelo teste $\mathrm{F}$, respectivamente. 
que os fatores em estudo explicaram, com boa confiabilidade, a absorção desses nutrientes.

Os modelos para as concentrações de nutrientes na folha diagnóstica (Figura 2) e de seus respectivos conteúdos na parte aérea das mudas (Figura 3) apresentam comportamento semelhante para a maioria dos nutrientes, exceto para $\mathrm{S}, \mathrm{Ca}, \mathrm{Zn}$ e Fe.

A adubação com superfosfato triplo (SFT) aumentou as concentrações de $\mathrm{S}$ e de $\mathrm{Ca}$ na folha (Figura 2a,b), provavelmente porque o fertilizante utilizado continha $2,0 \%$ de $\mathrm{S}$ e $16,4 \%$ de $\mathrm{Ca}$, favorecendo sua absorção e armazenamento na folha diagnóstica; por outro lado, reduziu o acúmulo total desses nutrientes na parte aérea da planta (Figura 3a,b), possivelmente porque as doses maiores de SFT restringiram o crescimento da parte aérea (Figura 1b). Todavia, deve-se considerar que a adição de $\mathrm{P}$ favorece a lixiviação de $\mathrm{S}$ e a precipitação de Ca, o que diminuiria o acúmulo total desses nutrientes ao final dos 150 dias de cultivo.
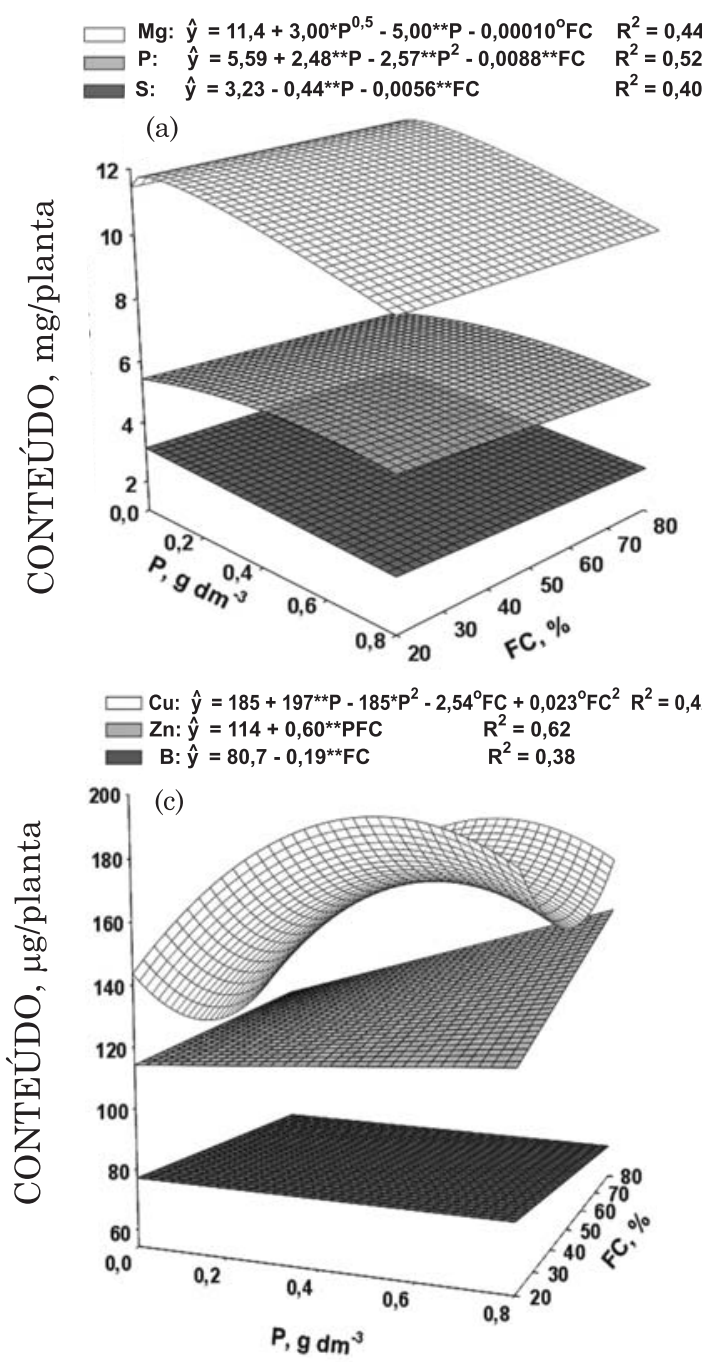

A adubação com SFT aumentou linearmente o conteúdo de $\mathrm{Zn}$ na parte aérea (Figura 3c), possivelmente devido ao fornecimento de $\mathrm{Zn}$ pelo próprio fertilizante, que continha $0,04 \%$ de Zn. Contudo, sua concentração na folha foi inicialmente reduzida, talvez em razão do efeito de diluição; posteriormente, aumentou e tornou-se mais expressivo com o aumento de $\mathrm{FC}$ no substrato, devido à interação significativa entre $\mathrm{P}$ e FC, o que pode ter elevado a concentração desse nutriente na folha (Figura 2c), por causa do menor crescimento da parte aérea das plantas nos substratos muito ricos em FC (Figura 1b).

A concentração de Fe na folha diagnóstica foi maior nos extremos das doses de P (Figura 2d), possivelmente devido ao efeito de concentração, visto que, nessa situação, a produção de MSPA foi menor (Figura 1b). Por sua vez, a variação no conteúdo de Fe na parte aérea foi expressiva, apresentando comportamento tipicamente quadrático para $\mathrm{P}$ e FC (Figura 3d), semelhante à produção de MSPA
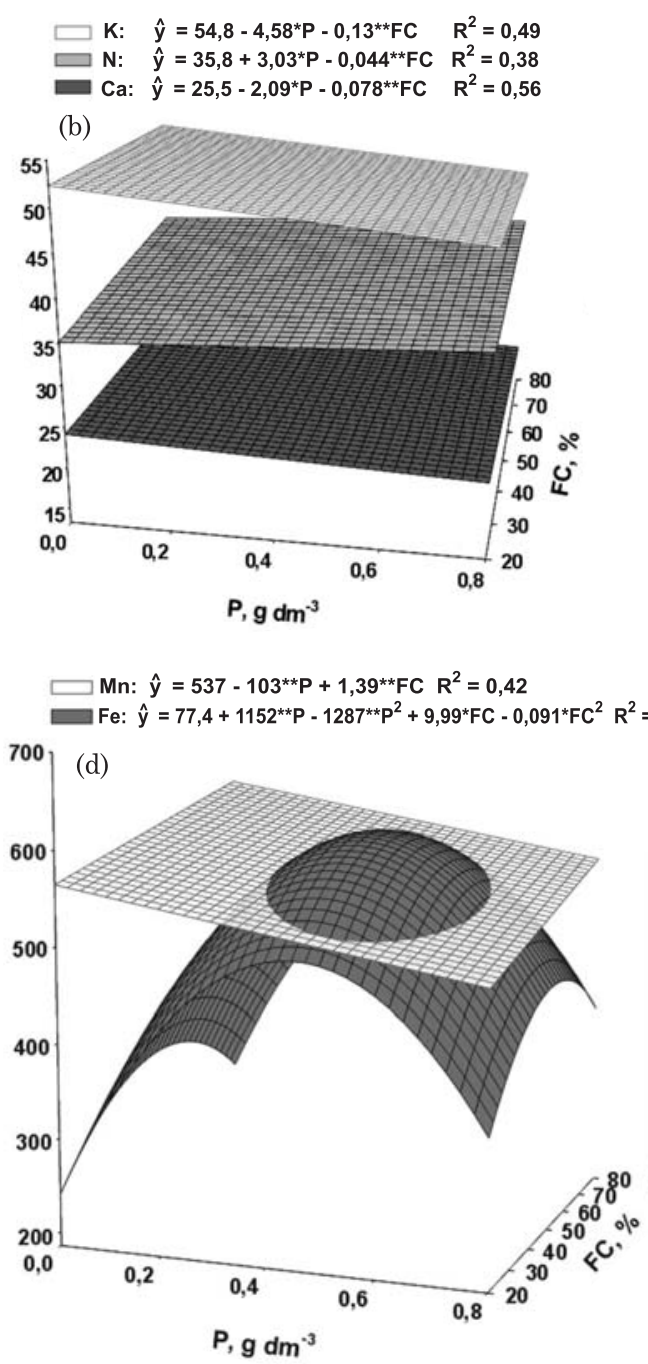

Figura 3. Conteúdo de nutrientes na parte aérea de mudas de cacaueiro, clone PH 16, aos 150 dias, em função da dose de $\mathrm{P}$ e da percentagem de fibra de coco (FC) no substrato: (a) Mg, P e S; (b) K, N e Ca; (c) $\mathrm{Cu}, \mathrm{Zn}$ e B; e (d) Mn e Fe. ${ }^{\circ},{ }^{*} \mathrm{e}^{* * *}$ : significativo a 10,5 e $1 \%$ pelo teste $\mathrm{F}$, respectivamente. 
(Figura 1b), porém com amplitude de variação bem superior. Esse comportamento pode ser devido tanto à produção de MSPA quanto à variação da concentração de $\mathrm{Fe}$ nos demais compartimentos da planta, em especial nas demais folhas, cuja amplitude foi de 150 a $910 \mathrm{mg} \mathrm{kg}^{-1}$ de Fe. Ao comparar esse limite superior com valores sugeridos como normais por Raij et al. (1997) e Malavolta (2006), pode-se concluir que esse nutriente deve estar em concentração tóxica - fato também constatado por Marrocos \& Sodré (2004).

A adubação com SFT, além de aumentar a absorção de $\mathrm{P}$ (Figuras 2a e 3a), aumentou também a concentração e o conteúdo de $\mathrm{Ne}$ Cu na planta (Figuras 2b, 2c, 3b e 3c), mas diminuiu a absorção de $\mathrm{Mg}, \mathrm{K} \mathrm{e}$ Mn (Figuras 2a,b,d e 3a,b,d). O aumento das doses de SFT favorece a lixiviação de Mg e K (Souza Jr. et al., 2008) e, provavelmente, esse foi o principal fator para justificar o menor acúmulo desses nutrientes na planta; contudo, não se pode esquecer que existe inibição competitiva do Ca, presente no SFT, com o Mg e o K (Malavolta, 2006). Para o Mn, há inibição não competitiva com o P (Malavolta, 2006), o que explicaria seu menor acúmulo na planta.

De modo geral, o aumento da proporção de FC no substrato reduziu a concentração e o conteúdo de nutrientes na planta (Figuras 2 e 3), exceto para os micronutrientes catiônicos. A disponibilidade natural pequena da maioria dos nutrientes e a CTC baixa da FC (Quadro 1) favoreceram o suprimento menor de nutrientes às plantas e a perda maior por lixiviação.

A FC é mais pobre em Fe (Quadro 1) e seu aumento da composição do substrato favoreceu a redução da concentração desse nutriente na folha, porém a magnitude dessa variação no modelo foi discreta (Figura 2d). No entanto, o seu conteúdo na planta apresentou efeito quadrático e variação ampla (Figura 3d), sugerindo que apenas a alteração do substrato não seria suficiente para eliminar possíveis problemas de toxidez de Fe. Fatores como pH e condições de oxirredução são determinantes na disponibilidade desse nutriente (Lindsay, 1979). Esses fatores podem auxiliar no entendimento da variação ampla da concentração e do conteúdo de Fe na planta, em especial porque o tubete é pequeno e a perfeita homogeneização dos substratos é difícil de ser conseguida, além de a lâmina de água efetiva que chega a cada tubete poder variar, o que influencia as condições de oxirredução.

A concentração e o conteúdo de Zn e de Mn na planta foram maiores com o aumento de FC no substrato (Figuras 2c,d, 3c,d), apesar de a FC ser mais pobre nesses nutrientes (Quadro 1). Esse resultado pode ser devido à menor disponibilidade de $\mathrm{P}$ nos substratos ricos em FC, que favoreceu a absorção de Zn e Mn pelas plantas, devido à inibição da absorção desses últimos nutrientes pelo $\mathrm{P}$ (Malavolta, 2006). Não se pode esquecer de que houve oito aplicações de mancozeb, fungicida/acaricida que contém $\mathrm{Zn}$ e Mn, o que pode ter influenciado na absorção desses nutrientes, e de que a disponibilidade de $\mathrm{Mn}$ depende das condições de oxirredução e pH do substrato (Lindsay, 1979) e sua absorção é inibida pelo $\mathrm{Fe}$ (Malavolta, 2006).

A época de adubação foi analisada por contraste; a aplicação de $\mathrm{P}$ em cobertura, aos 30 dias após o estaqueamento, em relação à adubação no preparo do substrato, não resultou em alteração significativa das variáveis de crescimento e nutricionais, exceto porque aumentou a concentração de $\mathrm{P}$ e reduziu a de $\mathrm{Zn}$ na folha diagnóstica e aumentou o conteúdo de $\mathrm{N}$ na parte aérea da muda.

\section{CONCLUSÕES}

1. A sobrevivência das mudas não foi alterada por substratos, doses ou épocas de aplicação de P.

2. A adição de $\mathrm{P}$ em cobertura, apesar de aumentar seu teor na folha, não resultou em incremento das variáveis de crescimento da muda de cacau.

3. As variáveis biométricas e nutricionais responderam à aplicação de $\mathrm{P}$ e à composição do substrato.

4. As proporções melhores de fibra de coco no substrato variaram de 30 a $55 \%$, em volume, e as doses recomendáveis de $\mathrm{P}$ no plantio variaram de acordo com o substrato, sendo o nível crítico foliar de $1,75 \mathrm{~g} \mathrm{~kg}^{-1}$ de P.

\section{LITERATURA CITADA}

ABAD, M.; FONTES, F.; CARRIÓN, C. \& NOGUERA, V. Physical properties of various coconut coir dusts compared to peat. HortScience, 40:2138-2144, 2005.

BARROS, N.F. \& NOVAIS, R.F. Eucalipto. In: RIBEIRO, A.C.; GUIMARÃES, P.T.G. \& ALVAREZ V., V.H., eds. Recomendação para o uso de corretivos e fertilizantes em Minas Gerais - $5^{\mathrm{a}}$ aproximação. Viçosa, MG, Comissão de Fertilidade do Solo do Estado de Minas Gerais, 1999. p.303-305.

BATAGLIA, O.C. \& FURLANI, P.R. Nutrição mineral e adubação para cultivos em substratos com atividade química. In: ENCONTRO NACIONAL SOBRE SUBSTRATO PARA PLANTAS, 4., 2004, Viçosa, MG. Anais... Viçosa, MG, Universidade Federal de Viçosa, 2004. p.106-125.

BAUMGARTEN, A. Methods of chemical and physical evaluation of substrates for plants. In: ENCONTRO NACIONAL SOBRE SUBSTRATO PARA PLANTAS, 3., Campinas, 2002. Anais...Campinas, Instituto Agronômico, 2002. p.7-15.

BRASIL. Instrução Normativa n.46, de 12 de setembro de 2006. Métodos analíticos oficiais para análises de substratos para plantas e condicionadores de solo. Diário Oficial da República Federativa do Brasil. Poder Executivo. Brasília, 14 de set. 2006. n.177, seção 1. p.2-3. 
De BOODT, M. \& VERDONCK, O. The physical properties of the substrates in horticulture. Acta Hortic., 26:37-44, 1972.

EMPRESA BRASILEIRA DE PESQUISA AGROPECUÁRIA EMBRAPA. Manual de análises químicas de solos, plantas e fertilizantes. Brasília, 1999. 370p.

FARIA, J.C. \& SACRAMENTO, C.K. Enraizamento e crescimento de estacas herbáceas do cacaueiro (clones CEPEC 42, TSH 516 e TSH 1188) em função da aplicação do ácido indolbutílico (AIB). R. Bras. Frutic., 25:192-194, 2003.

GONÇALVES, J.L.M.; RAIJ, B.van \& GONÇALVES, J.C. Florestais. In: RAIJ, B.van; CANTARELLA, H.; QUAGGIO, J.A. \& FURLANI, A.M.C., eds. Recomendação de adubação e calagem para o estado de São Paulo. 2.ed. Campinas, Instituto Agronômico de Campinas, 1997. p.247-259. (Boletim Técnico, 100)

LINDSAY, W.L. Chemical equilibria in soils. New York, John Wiley \& Sons, 1979. 449p.

MACKAY, A.D. \& BARBER, S.A. Effect of soil moisture and phosphate level on root hair growth of corn roots. Plant Soil, 86:321-331, 1985.

MALAVOLTA, E. Manual de nutrição mineral de plantas. São Paulo, Ceres, 2006. 631p.
MARROCOS, P.C. \& SODRÉ, G.A. Sistema de produção de mudas de cacaueiros. In: ENCONTRO NACIONAL SOBRE SUBSTRATO PARA PLANTAS, 4., Viçosa. MG, 2004. Anais... Viçosa, MG, Universidade Federal de Viçosa, 2004. p.283-311.

RAIJ, B.van; CANTARELLA, H. \& QUAGGIO, J.A. Estimulantes. In: RAIJ, B.van; CANTARELLA, H.; QUAGGIO, J.A. \& FURLANI, A.M.C., eds. Recomendação de adubação e calagem para o estado de São Paulo. 2.ed. Campinas, Instituto Agronômico de Campinas, 1997. p.9395. (Boletim Técnico, 100)

SONNEVELD, C.; van der ENDE, J. \& van DIJK, P.A. Analysis of growing media by means of a 1:1 $1 / 2$ volume extract. Comm. Soil Sci. Plant Anal., 5:183-202, 1974.

SOUZA JR., J.O.; CARMELLO, Q.A.C. \& FARIA, J.C. Características químicas do lixiviado na fase de enraizamento de estacas de cacau em substratos adubados com fósforo. R. Bras. Ci. Solo, 32:1573-1581, 2008.

WILLIAMS, K.A. \& NELSON, P.V. Modifying a soilless root medium with aluminum influences phosphorus retention and Chrysanthemum growth. HortScience, 31:381-384, 1996. 